Original Research Paper

\title{
Comparative Analysis of Various Diagnostic Methods for Trichomonas Vaginalis Infection in Trinidad and Tobago
}

\author{
${ }^{1}$ Aruna Kumari Divakaruni, ${ }^{1}$ Bisram Mahabir, ${ }^{2}$ FA Orrett, ${ }^{3}$ Sneha Rao A, \\ ${ }^{4}$ Srikanth Adidam, ${ }^{3}$ Vijay Kumar Chattu and ${ }^{3}$ AV Chalapathi Rao \\ ${ }^{1}$ Consultant, Queens Park Counseling Center and Clinic, Ministry of Health, Port of Spain, Trinidad And Tobago \\ ${ }^{2}$ Consultant, South West Regional Health Authority, Trinidad And Tobago \\ ${ }^{3}$ Faculty of Medical Sciences, The University of the West Indies, Trinidad And Tobago \\ ${ }^{4}$ North West Regional Health Authority, Port of Spain, Trinidad And Tobago
}

Article history

Received: 15-04-2018

Revised: 28-06-2018

Accepted: 06-08-2018

Corresponding Author:

Vijay Kumar Chattu

Public Health and Primary

Care, The University of the

West Indies, Trinidad And

Tobago

Email: vijay.chattu@sta.uwi.edu

\begin{abstract}
Trichomonas vaginalis infection is a common sexually transmitted infection in the reproductive age group, which may lead to complications. Wet mount examination is the most common test for diagnosis, but it has low sensitivity. Fluorescent antibody staining can be used for diagnosis, but it is expensive and requires special microscopic facility. Culture is considered as the gold standard, but it takes a long time for diagnosis and not practical for routine use. OSOM Trichomonas Rapid Test is a recently introduced rapid method based on immunochromatographic assay of trichomonal protein antigens. Nucleic Acid Amplification Tests (NAAT's) are highly sensitive, it requires expensive resources and is currently not widely available in developing countries. The present study was done to compare these four available diagnostic techniques for detection of trichomoniasis. Samples were collected from a total of six hundred ninety two (692) patients. Wet mount examination, Fluorescent antibody staining, InPouch culture and OSOM Trichomonas Rapid Test were performed. Out of 692 patients included in our study, Four hundred and twenty two (422) were females and Two hundred seventy (270) were males. 12.4\% positive cases of Trichomoniasis were detected by InPouch culture. OSOM Trichomonas Rapid Test detected in (11.2\%) cases and Fluorescent antibody staining detected in $9.3 \%$ cases, whereas, wet mount examination detected only $3.5 \%$ positive cases. OSOM Trichomonas Rapid Test showed sensitivity and specificity of $90.5 \%$ and $98.5 \%$, respectively. Comparative analysis of four diagnostic methods indicates OSOM Trichomonas Rapid Test is a easily performed simple test with higher sensitivity and specificity than both wet mount examination and Fluorescent antibody staining. Standardized strips of high quality with reasonable costing could be recommended as a routine test in clinic as well as in rural settings.
\end{abstract}

Keywords: Fluorescent Antibody Staining, InPouch Culture, OSOM Trichomonas Rapid Test, Trichomonas vaginalis, Wet Mount Examination, Nucleic Acid Amplification Tests (NAAT's)

\section{Introduction}

Trichomonas vaginalis infection is a common Sexually Transmitted Infection (STI) in the reproductive age group, which needs to be promptly and accurately diagnosed to prevent complications. According to the World Health Organization (WHO, 2008) estimates that more than 276 million cases of trichomoniasis occur annually worldwide. The estimated incidence in normal population is approximately $10 \%$. Eight (8) million new cases are reported annually in North America. According to the World Health Organization, almost half of all curable sexually transmitted diseases worldwide might be attributable to $T$ vaginalis (Bickley et al., 1984).
Science

Publications 
Estimates of the prevalence of $T$ vaginalis infection in the United States range between $8.6 \%$ and $47 \%$ for women and between $2.8 \%$ and $17 \%$ for men (Schwebke, 2004). However, prevalence estimates still vary widely even when reviewing data that use similar diagnostic methods, since the observed populations are not representative of the general population (Maureen et al., 2008). Diagnosis of the condition is difficult, since the symptoms of trichomoniasis mimic those of other STI's and detection methods lack precision. The diagnosis is made by different methods, which include: (a) Wet mount study, (b) Pap smear study, (c) Rapid tests, (d) Direct fluorescent antibody test, (e) Culture and (f) Molecular techniques. A number of studies have shown that there are differences in the sensitivity and specificity ranging from $30-95 \%$ for these tests.

Currently available screening tests tend to lack sensitivity. Culture is considered to be gold standard for the diagnosis of trichomoniasis is not widely available and it is time consuming. Although Nucleic Acid Amplification Tests (NAAT's) are highly sensitive, it requires expensive resources and NOT widely available in developing countries (Kurth et al., 2004). New rapid strip tests that are available are simple to use and their performance has been indicated to be satisfactory.

There are no well-established reports about prevalence and evaluation of diagnostic tests of this disease in Trinidad and Tobago. A few vague reports from Queen's Park Counseling Centre and Clinic (QPCC\&C) were available suggesting 1 to $2 \%$ prevalence (diagnosed only by wet mount preparation). Diagnosis of the condition is difficult since the symptoms of trichomoniasis mimic those of other STI's and detection methods lack precision. There are no published reports about prevalence of this disease in the Caribbean Islands. Therefore, it is important to study the most reliable and cost effective methods with reasonable turnaround times for the diagnosis and better management of the patients at our institutions.

The purpose of the study was to compare various available tests feasible in our current setting for diagnosis of Trichomoniasis while highlighting their performance characteristics, advantages and limitations.

\section{Patients and Methods}

This was a cross-sectional study. Patients were recruited at the public S.T.I clinic (QPCCC), Ministry of Health, Trinidad and Tobago. All males and females were eligible to be included if they were over 15 years of age or had sexual exposure and consented for routine genital examination. Experienced Nurses, Nursing Assistants, Doctors and support staff were recruited for the study. Informed consent was obtained from all the patients recruited in the study. In the case of minors (persons below the age of eighteen), informed consent was obtained from the parent or guardian. At the time of obtaining consent the participant was given the required information regarding the purpose of the study, confidentiality and rights and responsibilities. A total of six hundred ninety two (692) patients were studied. Four hundred and twenty two (422) were females and Two hundred seventy (270) were males. Sample size was determined based on the annual patient attendance at the clinic and estimated prevalence of trichomoniasis at the clinic.

A questionnaire that was prepared, tested, revised and approved by Medical Ethics Committee was administered to collect the clinical data. Following a routine clinical examination, the samples were collected for testing.

Four (4) Vaginal/Urethral swabs were collected- one for wet mount preparation of T.V; and one for In Pouch culture; and one each for OSOM Rapid test/and Fluorescent antibody testing. Cotton swabs and Dacron swabs were used for the collection of samples. The samples were then tested using various methods, which are based on different principles as described below.

\section{Wet Mount Preparation}

It was done at the test site immediately by using one drop of normal saline mixed with swab secretions on the glass slide. The wet mount preparation was studied at $\mathrm{x}$ 100 for motile organisms and at $\mathrm{x} 400$, where ever required confirmation. $T$ vaginalis was readily identified by its pear shape, five flagella and twitching motion (darting between non-motile cells).

\section{OSOM Trichomonas Rapid Test}

It is an immunochromatographic assay that detects antigens of the causative organism as shown in Fig. 1. The dipstick method uses color immunochromatographic capillary flow technology. The solubilized Trichomonas proteins from a vaginal swab mixed in sample buffer is tested using the OSOM Trichomonas Rapid Test stick. Trichomonas vaginalis when present in the sample forms a complex with the primary anti-Trichomonas antibody conjugated to colored particles (blue). A second antiTrichomonas Antibody will then bind to the complex. A visible blue test line along with the red control line will indicate a positive result. Internal controls were used to aid in determining the test validity and also culture positive and negative samples were used as controls. The steps in the test procedure includes: (1) To add sample buffer to each test tube, (2) Mix and soak the swab in buffer for one minute, (3) Squeeze liquid from swab into the buffer solution and discard the swab in a suitable biohazardous waste container. (4) Dip the OSOM test stick with the absorbent end in the sample buffer, (5) Read the results and (6) discard the test tubes and test stick. 


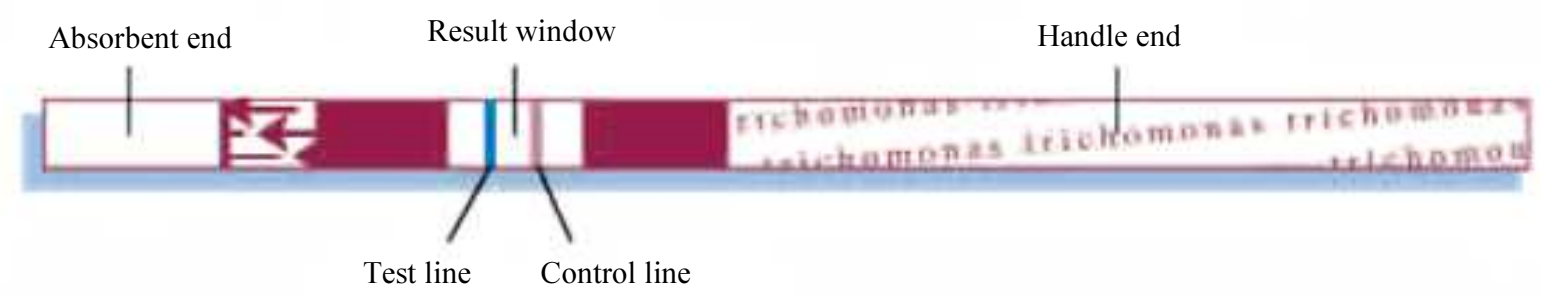

Fig. 1: Trichomonas OSOM Rapid Test Strip

\section{Interpretation of Results}

A blue test line and a red control line is a positive result. Non-appearance of blue line indicates that the test is negative for Trichomonas antigens.

\section{Limitations}

i. The OSOM ${ }^{\circledR}$ Trichomonas Rapid Test is only for the qualitative detection of $T$. vaginalis antigen from vaginal swabs and the saline solution remaining from a wet mount of a vaginal swab

ii. The performance of the $\mathrm{OSOM}^{\circledR}$ Trichomonas Rapid Test with specimens other than vaginal fluid or the saline solution remaining from a wet mount of a vaginal swab has not been established

iii. The results obtained with this kit yield data that must be used only as an adjunct to other information available to the physician

iv. This test does not differentiate between viable and non-viable organisms

v. This test does not differentiate between individuals that are carriers and individuals that have an acute infection

vi. Patients with vaginitis/vaginosis symptoms may have mixed infections. Therefore a test indicating the presence of $T$. vaginalis does not rule out the presence of Candida vulvo-vaginitis or bacterial vaginosis

vii. A negative result may be obtained if the specimen collection is inadequate or if antigen concentration is below the sensitivity of the test. A negative OSOM ${ }^{\circledR}$ Trichomonas Rapid Test result may warrant additional patient follow up

viii. Women with vaginal discharge should be evaluated for risk factors of cervicitis and pelvic inflammatory disease and for other organisms including Neisseria gonorrhea and Chlamydia trachomatis

ix. Samples contaminated with preparations containing iodine or by the immediate prior use of vaginal lubricants are not recommended

x. Staphylococcus aureus in specimens at concentrations higher than $1 \times 10^{8}$ organisms per $\mathrm{mL}$ may interfere with the test results in negative samples. These concentrations of $S$. aureus are higher than would be expected to be present in normal patient samples

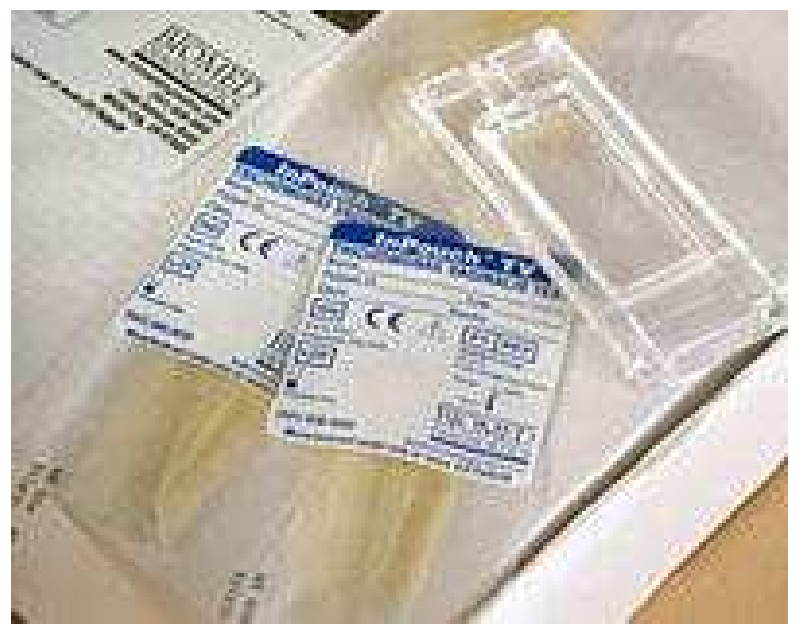

Fig. 2: InPouch culture system: Plastic pouch and microscopic observation chamber

\section{InPouch T.V Culture System}

It is a self-contained system for the detection of T.V as shown in Fig. 2 developed by Biomed Diagnostics, Santa Clara, California. The medium is selective for transport and growth of T.V while inhibiting the growth of contaminating micro-organisms. The InPouch consists of high barrier oxygen resistant plastic pouch with two (2) "V" shaped chambers. Specimen collection and incubation of "InPouch" media were done according to manufacturer's description. All precautionary measures were well observed. Microscopic observation of the chamber is done with or without using plastic microscope viewing clip. Transport and off-site testing can be performed easily due to the flexible packaging and integral design of the InPouch. An inoculum containing 1 to 10 Trichomonads is sufficient to cause positive test.

The specimens collected for InPouch T.V culture testing were transported to microbiology laboratory attached to the University of the West Indies within two (2) hours. The culture pouch was incubated at $37^{\circ} \mathrm{C}$ and the results were read at $24 \mathrm{hrs}$ and alternate days for a maximum of five (5) days. The results were read independent of wet mount or rapid test. 
Fluorescent Antibody test (indirect fluorescent antibody method)-Fluorescent antibody Reagent contains murine monoclonal antibody specific for Trichomonas. The reagent is formulated with Evans Blue Counter stain in a protein stabilized buffer solution containing $0.1 \%$ sodium azide as a preservative. Specimens were collected and smears were prepared on charged or activated slides. These slides were fixed in $10 \%$ acetone solution for $2 \mathrm{~min}$ and later washed in distilled water. The smears fixed in acetone were washed twice with Phosphate Buffered Saline (PBS). Standard Indirect Fluorescent Antibody (IFA) procedures were used. The smears were pretreated with goat serum were incubated with diluted antibody serum $(1: 20)$ for $30 \mathrm{~min}$ at room temperature. Slides were washed with PBS $(2 \times 15 \mathrm{~min})$, air dried and re-incubated for 30 minutes with fluorescein-labeled immunoglobulin (Trichomonas vaginalis (7111) Antibody from Santa Cruz Biotechnology, Inc). Slides were washed in PBS $(3 \times 10$ min) and then mounted with Immuno-mount. The stained slides were read under incident Immunofluorescence microscope. Apple green fluorescence- marginal or general was considered positive. Positive controls were prepared from culture positive medium and negative controls were prepared from known negative samples.

All these four diagnostic methods were compared with each other for technology, sensitivity, specificity, cost effectiveness and reliability. Culture method InPouch was considered as "gold standard".

Based on the results of these tests, if any one of the tests becomes positive for Trichomonas Vaginalis, patients were treated according the CDC guidelines. Sensitivity and Specificity were calculated using standard formula and Culture results were used as a reference and "Gold Standard":
Sensitivity: Is the ability of a test to correctly classify an individual as 'diseased'

$=$ (true positive $) /($ true positive + false negative)

$=$ Probability of being test positive when disease present.

Specificity: The ability of a test to correctly classify an individual as disease-free is called the test's specificity.

Specificity $=($ true negative $) /($ true negative + false positive)

$=$ Probability of being test negative when disease absent.

Results were documented and statistical analysis was done with the help of S.P.S.S Software. The p-value is significant at $<0.05$.

\section{Results}

In the present study, a total of six hundred ninety two (692) patients were included and out of the total number of cases, four hundred and twenty two (422) were females and Two hundred seventy (270) were males.

Various laboratory tests were done in the evaluation of validity, efficacy and usability in our laboratory conditions. The results of which are shown below in Fig. 3. The results show that positivity was $3.5 \%$ with wet mount as compared $>12 \%$ in the other two methods-OSOM Rapid test and InPouch culture. Immunofluorescent (IF) method was done in 232 patients and was found to be less sensitive (9.3\%) compared to the reference -InPouch method.

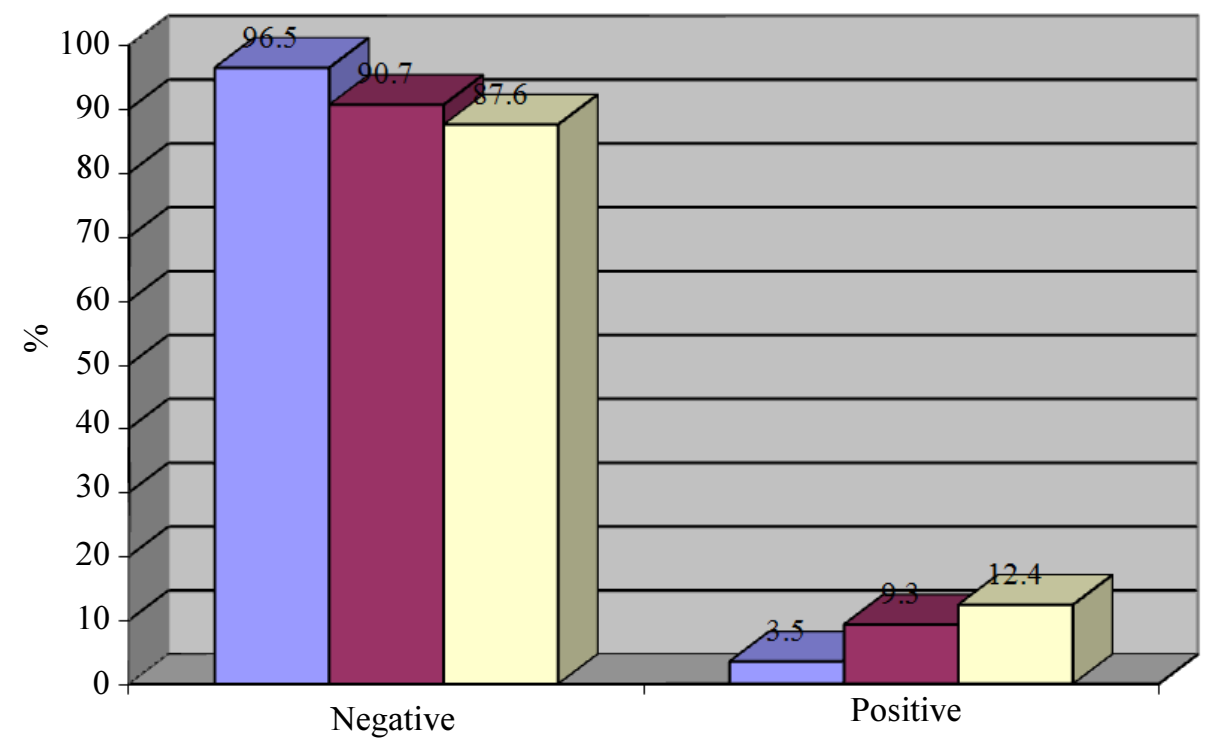

Fig. 3: Comparison of laboratory test results
Wet Mount

Rapid Test

$\square$ InPouch culture 
Table 1: Evaluation of testing methods in Trichomoniasis

\begin{tabular}{|c|c|c|c|c|c|c|c|c|c|}
\hline \multirow{2}{*}{$\begin{array}{l}\text { Test name } \\
\text { (no. of tests) }\end{array}$} & \multicolumn{2}{|c|}{ Sensitivity } & \multicolumn{2}{|c|}{ Specificity } & \multicolumn{4}{|c|}{ Predictive value } & \multirow{2}{*}{$\begin{array}{l}\text { Accuracy } \\
\%\end{array}$} \\
\hline & $\%$ & $95 \% \mathrm{CI}$ & $\%$ & $95 \% \mathrm{CI}$ & Pos & $95 \% \mathrm{CI}$ & Neg & $95 \% \mathrm{CI}$ & \\
\hline WET MOUNT (692) & 27.0 & $17.6-36.4$ & 99.8 & $99-100$ & 95.8 & $95-96.6$ & 90.5 & $88.3-92.7$ & 90.75 \\
\hline OSOM Rapid test (689) & 90.5 & $84.4-96.6$ & 98.5 & $97.5-99.5$ & 89.5 & $83.2-95.8$ & 98.6 & $97.8-99.4$ & 97.50 \\
\hline Immunofluorescent test (232) & 75.0 & $61-89$ & 96.4 & $93.7-99.1$ & 79.4 & $65.7-93.1$ & 95.4 & $92.4-98.4$ & 93.10 \\
\hline
\end{tabular}

Overall OSOM rapid test performance with regards to accuracy, positive and negative predictive value are better than the wet mount and IF method

The comparison of various tests with regards to sensitivity, specificity, Predictive value and accuracy are shown in Table 1. The results were compared against InPouch Culture method used as a reference method. Sensitivity of wet mount method was $27 \%$ compared to $90.5 \%$ and $75 \%$ for OSOM Rapid test and IF method respectively. Specificity was high with wet mount (99.8\%) compared to $89.5 \%$ and $79.4 \%$ with OSOM Rapid test and IF method respectively.

Overall OSOM rapid test performance with regards to accuracy, positive and negative predictive value is better than the wet mount and IF method.

\section{Discussion}

Clinical signs and symptoms of $T$ vaginalis infection in women and men are neither sensitive nor specific enough to be used for diagnosis. Therefore, positive identification of the organism is required (Schwebke, 2004; Garber, 2005; Kurth et al., 2004). The disease can be difficult to diagnose due to its heterogeneous presentation and limitations, of currently available diagnostic tests that are fraught with imperfections. Insensitive traditional methods of detection greatly underestimate the prevalence of Trichomonas vaginalis.

We compared wet mount preparation, OSOM rapid test and Immunofluorescent test against InPouch culture, which was used as a standard reference method. The newer InPouch method is considered to be advantageous due to simplicity, high sensitivity and specificity and cost-effectiveness (Sood et al., 2007). Culture is the "gold standard"/reference test for the diagnosis of trichomoniasis (Kurth et al., 2004) Although studies recommended for widespread use of the test, (Sood et al., 2007) it is not widely available and specimens must be examined for up to 7 days until being considered negative. This method also requires a laboratory and specialized equipment for growing and identifying $T$. vaginalis. It is not a practical diagnostic method in sexually transmitted disease clinics, adult health centers and physicians' offices, where there is a rapid turnaround of patients (Kurth et al., 2004).

The sensitivity of wet mount preparation was quite low $(27 \%, 95 \%$ CI $17.6-36.4)$ and is lower than reported in some studies. Although the specificity is $99.8 \%$ and direct microscopic examination of the vaginal specimen remains the most widely utilized diagnostic test for this infection, it is clearly evident that it is an unsatisfactory and unreliable screening test. Low sensitivity compared to culture has been reported by a number of studies (Seña et al., 2007; Jatau et al., 2006). The reasons for low sensitivity include: The identification of trichomonas using a wet mount slide depends on: (1) concentration of the organism and the expertise and experience of the examiner (Lobo et al., 2003). (2)The size of the Trichomonad is approximately the same as that of a lymphocyte (10 to $20 \mu \mathrm{m})$ or a small neutrophil; when not motile, a Trichomonad can be difficult to differentiate from the nucleus of a vaginal epithelial cell and (3) Motility is very dependent on the temperature of the specimen.

In our present study we found that the OSOM rapid test had the sensitivity of $90.5 \%(95 \% \mathrm{CI}=84.4-96.6)$ and specificity of $98.5 \%(95 \% \mathrm{CI}=97.5-99.5)$. These results were comparable to other studies. One of studies reported the following results: OSOMT rich rapid test had very good performance, with sensitivity, specificity, efficiency, positive predictive value and negative predictive value of $94.7,100,99.9,100$ and $99.9 \%$, respectively (Campbell et al., 2008). The implementation of OSOM Trich rapid test for diagnosing $T$. vaginalis may be recommended for use in the routine diagnosis. A rapid diagnostic test that does not require special equipment or a formal laboratory is needed in these settings and can be performed on site by laboratory personnel, nurses and medical practitioners with minimal training and it would also decrease labor costs. It is important to ensure that the given batch of strips is within expiration date and passes quality control test. Rapid point-of-care test is needed where microscopy and culture are impractical (Kurth et al., 2004; Razia et al., 2015).

Immunofluorescent test using specific antibodies against Trichomonas vaginalis was found to be more sensitive $(75 \%, 95 \% \mathrm{CI}=61-89)$ than wet preparation. Specificity and accuracy was above $90 \%$. The results compare well with other studies (Seña et al., 2007; Krieger et al., 2003; Uneke et al., 2007). However, the results of the test were not superior to Rapid test. The test requires expensive antibodies, an elaborate procedure, a fluorescent microscope and a trained staff to interpret the results. 


\section{Costing Exercise}

Comparison of costs/test for different methods was performed. Inpouch culture was TT $\$ 24 /-$ or (US\$ 4); OSOM rapid test - TT $\$ 80 /-$ or (US\$13); IF test $=$ TT $\$ 70 /-$ or (US\$12) and Wet mount costs TT\$10 (approx.) which is the cheapest of all the four tests. The Rapid test was found to be more expensive than the other methods. Labour cost and time factor are not included in these calculations.

Nucleic Acid Amplification Tests (NAAT's) being highly sensitive, have the potential to replace currently evaluated diagnostic tests, provided that it is less resource intense and can be applied at all health care settings and in rural areas.

\section{Conclusion}

Trichomonas vaginalis is a common STI in the reproductive age group which needs to be promptly and accurately diagnosed to prevent complications. Although wet mount examination is a commonly used methods for diagnosis of $T$. vaginalis infection, but it is less sensitive and needs microscope and technical expertise. Fluorescent antibody test is expensive and requires laboratory resources. Culture is impractical for routine use due the nature of the test and better serves as a reference test to evaluate diagnostic methods. Based on the present study, OSOM rapid strip test is a feasible and practical alternative to implement for routine testing in STI clinics and also field setting. The potential of this test being widely introduced depends on maintaining quality and standards of the product and availability at a subsidized price. It is a rapid diagnostic test that does not require special equipment or a formal laboratory is needed. OSOM Trichomonas Rapid Test had a good sensitivity and being a point of care test it is useful in remote areas lacking microscopic and culture facilities. Moreover the rapid tests suits for settings to decrease labor costs as well as to provide test results on the same day so that the patients are put on treatment without loss to followup thereby decreasing the chance of spreading the infection to others. The potential of Nucleic Acid Amplification Tests (NAAT's) being highly sensitive test to replace currently evaluated diagnostic tests is a possibility, provided that it is less resource intense, cost-effective and can be applied at all health care settings including rural areas.

\section{Acknowledgement}

We sincerely thank National AIDS Coordination Committee for supporting and funding the research project. We are grateful for the participation of patients, clinicians and laboratory staff of Queens Park Counseling Center and Clinic, Ministry of Health, Port of Spain, Trinidad. We thank Mr. Steve Lalman at The University of the West Indies, St. Augustine for providing laboratory support.

\section{Conflict of Interest}

The authors have no conflict of interest financially or otherwise.

\section{Author's Contributions}

Aruna Kumari Divakaruni: Research execution, logistics, development of questionnaire and data collection.

Bisram Mahabir: Administrative support, Ethical approvaland expert advice.

FA Orrett: Lab investigations in Microbiology.

Sneha Rao A: Prepare references, editing and proof reading.

Srikanth Adidam: Review of literature.

Vijay Kumar Chattu: Manuscript preparation and editing.

AV Chalapathi Rao: Conception, design, interpretation and analysis of results.

\section{Ethics}

The study was approved bythe Ethics Committee (Research), Port of Spain General Hospital, Trinidad and Tobago.

\section{References}

Bickley, L.S., K.K. Krisher, A. Punsalang Jr., M.A. Trupei and R.C. Reichman et al., 1984. Comparison of direct fluorescent antibody, acridine orange, wet mount and culture for detection of Trichomonas vaginalis in women attending a public sexually transmitted diseases clinic. Sex Transm. Dis., 16: 127-31. PMID: 2479110

Campbell, L., V. Woods, T. Lloyd, S. Elsayed and D.L. Church, 2008. Evaluation of the OSOM trichomonas rapid test versus wet preparation examination for detection of Trichomonas vaginalis in specimens from women with a low prevalence of infection. J. Clin. Microbiol., 46: 3467-3469.

DOI: $10.1128 /$ JCM.00671-08

Garber, G.E., 2005. The laboratory diagnosis of Trichomonas vaginalis. Can. J. Infect. Dis. Med. Microbiol., 16: 35-38. DOI: 10.1155/2005/373920

Jatau, E.D., O.S. Olonitola and A.T. Olaynika, 2006. Prevalence of trichomonas infection among women attending antenatal clinics in Zaria, Nigeria. Annals African Med., 5: 178-181.

Krieger, J.N., M.R. Tam, C.E. Stevens, I.O. Nielsen and J. Hale et al., 2003. Diagnosis of trichomoniasis. Comparison of conventional wet-mount examination with cytologic studies, cultures and monoclonal antibody staining of direct specimens. Cytopathology, 14: 94-95. 
Kurth, A., W.L.H. Whittington, M.R. Golden, K.K. Thomas and K.K. Holmes et al., 2004. Performance of a new, rapid assay for detection of Trichomonas vaginalis. J. Clin. Microbiol., 42: 2940-2943. DOI: 10.1128/JCM.42.7.2940-2943.2004

Lobo, T.T., G. Feijo, S.E. Carvalho, P.L. Costa and C. Chagas et al., 2003. A comparative evaluation of the Papanicolaou test for the diagnosis of trichomoniasis. Sex Transm. Dis., 30: 694-699. DOI: 10.1097/01.OLQ.0000079525.04451.05

Maureen, M., Y. Liao, A.M. Gomez, C.A. Gaydos and D. D'Mellow, 2008. Factors associated with the prevalence and incidence of Trichomonas vaginalis infection among African American Women in New York City who use drugs. J. Infect. Dis., 197: 503-509. DOI: $10.1086 / 526497$

Razia, K., J. Noor, A. Siraj, K.H. Manzoor and R. Tamkin, 2015. Comparison of four diagnostic techniques for detection of Trichomonas vaginalis infection in females attending tertiary care hospital of North India. Indian J. Pathol. Microbiol., 58: 36-39. DOI: 10.4103/0377-4929.151172
Schwebke, J.R., 2004. Trichomoniasis care today: A clinician's guide to timely diagnosis and successful treatment. Genzyme Monograph.

Seña, A.C., W.C. Miller, M.M. Hobbs, J.R. Schwebke and P.A. Leone et al., 2007. Trichomonas vaginalis infection in male sexual partners: Implications for diagnosis, treatment and prevention. Clin. Infect. Dis., 44: 23-25. DOI: 10.1086/511144

Sood, S., S. Mohanty, A. Kapil, J. Tolosa and S. Mittal, 2007. InPouch TV culture for detection of Trichomonas vaginalis.. Indian J. Med. Res., 125: 567-571. PMID: 17598943

Uneke, C.J., M.N. Alo, O. Ogbu and D.C. Ugwuoru, 2007. Trichomonas vaginalis infection in human immunodeficiency virus-seropositive Nigerian women: The public health significance. Online J. Health Allied Sci., 6: 1-7.

WHO, 2008. Global incidence and prevalence of selected curable sexually transmitted infections2008. Geneva: World Health Organization; 2012. 